\title{
A complete general valence force field for secondary chlorides
}

\author{
W. H. Moore and S. KrIMM \\ Harrison M. Kandall Laboratory of Physics, University of Michigan, \\ Ann Arbor, Michigan 48104, U.S.A.
}

(Received 18 January 1973)

\begin{abstract}
A general valence force field for secondary chlorides has been refined which includes torsional force constants based on assignments of low frequency modes in model compounds. This force field also incorporates force constants related to observed intermolecular interactions. The good agreement between observed and calculated frequencies indicates that this force field will be useful in studying the conformation of secondary chlorides.
\end{abstract}

\section{INTRODUCTION}

IN AN effort to gain insight into the structure of stereo-specific poly(vinyl chloride), considerable attention has been given to the study of the vibrational frequencies of chlorine-substituted derivatives of the normal paraffins [1-6]. The well-established correlation in secondary chlorides between the frequency of the carbon-chlorine stretching vibration and the rotational isomer, and the availability of general valence force fields for hydrocarbons [7] and primary chlorides [8], formed the bases for the development of the original valence force field for secondary chlorides [1]. The lack of far infrared data, however, made it impossible to include force constants for the calculation of torsional modes in the refinement procedures used to develop these force fields. Relevant far infrared and Raman data for some model secondary chloride molecules are now available. We have already reported the assignment of these spectra, and have developed the force field necessary for the calculation of vibrations due to internal rotation [9]. We report here a complete general valence force field for secondary chlorides.

The spectral region of $\mathrm{C}-\mathrm{Cl}$ stretching in secondary chlorides, $500-700 \mathrm{~cm}^{-1}$, is highly diagnostic of local structure, because this vibration is relatively intense, is sensitive to the type of atoms trans to the chlorine atom across adjacent $\mathrm{C}-\mathrm{C}$ bonds, is sensitive to the geometry of the carbon chain, and takes place in a spectral region which except for the $\mathrm{C}-\mathrm{Cl}$ stretching vibrations themselves is relatively clear of other absorptions. The notation used here to describe molecular geometry relative

[1] C. G. OPaskar and S. KRIMM, Spectrochim. Acta 23A, 2261 (1967).

[2] T. Shumanouchi and M. Tasumi, Spectrochim. Acta 17, 755 (1961).

[3] P. Klaboe, Spectrochim. Acta 26A, 87 (1970).

[4] M. Tasumi and T. Shimanodchi, Polymer J. 2, 62 (1971).

[5] B. Schneider, J. StTokr, D. Doskočilova, S. SÝrora, J. JAKeš, and M. KolínskÝ, J. Polymer Sci. 28C, 1073 (1969).

[6] D. Doskočrlová, J. Ștokr, B. Schnemder, H. Pivcová, M. Kolf́nský, J. Petránek and D. Lím, J. Polymer Sei. 160, 215 (1967).

[7] R. G. SNyder and J. H. SchachtschneIder, Spectrochim. Acta 21, 169 (1965).

[8] R. G. SNyder and J. H. SohaChtschneider, J. Mol. Spectry 30, 290 (1969).

[9] W. H. Moone, J. H. C. Ching, A. V. R. WARRIER and S. KRIMM, Spectrochim. Acta 29A, 1847 (1974). 
to the chlorine atom or atoms is that suggested by Doskočrlova et al. [6]. This notation parallels that of SHIPMAN et al. [10]: an $S$ for secondary chlorines with subscripts to designate the type of atom trans to the chlorine and primes on these subscripts to indicate rotation away from the planar zig-zag conformation. However, instead of the $S_{\mathrm{HC}}$ or $S_{\mathrm{HH}}$ notation, we now use ${ }_{\mathrm{H}} S_{\mathrm{C}}$ or ${ }_{\mathrm{H}} S_{\mathrm{H}}$. The left hand subscript is used to describe the geometry to the immediate left of the chlorine atom. Similarly, the right hand subscript describes the molecular geometry to the right of the chlorine atom. In the simpler secondary chlorides, e.g. 2-chloropropane, 2-chlorobutane, and 3-chloropentane, the old notation is certainly adequate. However, in the tri-substituted heptanes this new formalism can be compounded to designate unambiguously the molecular conformation with respect to all chlorine atoms present, e.g. ${ }_{\mathrm{H}} S_{\mathrm{HH}} S_{\mathrm{HC}} S_{\mathrm{H}}$. When tetrahedral geometry is assumed, any rotation around a given carbon-carbon bond is meant to imply a rotation of $\pm 120^{\circ}$ from the planar zig-zag conformation. Using the notation described above, the empirically determined $\mathrm{C}-\mathrm{Cl}$ stretching frequencies can be assigned as a function of molecular geometry in the neighborhood of the chlorine atom [10].

Recently, on the basis of a study of rotational isomeric structures of monohalidesubstituted derivatives of $n$-pentanes and n-octanes, Gates et al. [11] stated that some of these assignments needed modification. Although an in-depth study of this work is only partially completed, from a comparison of these results with those reported by BENEDETTI and CECCHI [12] on 2-iodobutane and with our studies of secondary chlorides, two conclusions can be reached: (1) the characteristic frequency of the $\mathrm{C}-X$ stretching vibration ( $X=$ chlorine, bromine, or iodine) when the halogen is adjacent to a methyl group (as in 2-halogeno-butane, or 2,4 dihalogenopentane) does differ somewhat from the characteristic frequency of this stretching vibration in molecules like 3-chloropentane, 3-chlorooctane, etc. in which the halogen atom is between two methylene groups (see section on 3 -chloropentane); (2) in going from chloro- to bromo- to iodo-substituted hydrocarbons, the region of $\mathrm{C}-X$ stretching moves from $500-700 \mathrm{~cm}^{-1}$ in the case of the chlorine-substituted derivatives to $480-580 \mathrm{~cm}^{-1}$ in the case of the iodine-substituted hydrocarbons. For 2chlorobutane the $\mathrm{C}-\mathrm{Cl}$ stretching modes contribute only relatively small amounts to the potential energy distribution of the $\mathrm{C}-\mathrm{C}-\mathrm{C}$ bending, $\mathrm{C}-\mathrm{C}-\mathrm{Cl}$ bending, and torsional modes. However, for 2 -iodobutane this is not so. In ${ }_{\mathrm{H}} S_{\mathrm{H}} 2$-iodobutane, C-I stretching contributes 31 per cent to the potential energy distribution of an absorption due mainly to bending at $265 \mathrm{~cm}^{-1}$. Moreover, in the ${ }_{\mathrm{B}} S_{\mathrm{C}}$ rotational isomer of 2-iodobutane, the $\mathrm{C}-\mathrm{I}$ stretching vibration contributes 57 per cent to the potential energy distribution of a similar bending mode at $270 \mathrm{~cm}^{-1}$ [12]. This indicates that the atomic motions differ considerably according to the particular halogen which is present. Therefore, the assignment of bands in this region through the correlation of frequency shifts for different halogenated paraffins is a risky business. We therefore still use the empirical data of SHIPMaN et al. [10] as a guide in the assignments of the fundamental vibrations of chlorinated hydrocarbons.

[10] J. J. Shupman, V. L. FolT and S. Krrmm, Spectrochim. Acta 18, 1603 (1962).

[11] P. N. Gates, E. F. Mooney and H. A. Wrutis, Spectrochim. Acta 23A, 2043 (1967).

[12] E. Benedettr and P. Cecchr, Spectrochim. Acta 28A, 1007 (1972). 


\section{Development of Force Field}

OPASKAR and KRIMM [1] previously reported a force field for secondary chlorides which predicted well most of the normal modes of a group of model secondary chloride compounds and related polymers. However, their force field did not include any torsional force constants. In this study, our aim was to develop a force field which predicted torsional frequencies as well as those due to all other vibrational modes, and which reproduced the intermolecular interaction reported by WARRIER and KRIMM [13]. Initially, our force field was a composite of the most recent potential function for hydrocarbons reported by SNYDER [14] and the force field for secondary chlorides developed by OPASKAR and KRIMM [1], together with provisions for new torsional force constants and additional elements required to calculate vibrational frequencies of intermolecular normal modes. This force field was used to temporarily assign all new far infrared and Raman data on 2-chloropropane, 2chlorobutane, and the two stereoisomers of 2,4-dichloropentane. The results of this study are reported in the previous paper [9].

In order to obtain agreement with the observed spectra, a small modification had to be made in the hydrocarbon part of the force field. In the vibrational spectrum of 2-chloropropane, there are two $\mathrm{CH}_{3}$ bending modes, one symmetric and one antisymmetric. The portion of the potential function which was transferred from the $n$-paraffins determines the calculated frequencies of these bending vibrations. The initial normal coordinate calculations on 2-chloropropane predicted the antisymmetric umbrella vibration to be of higher frequency than the symmetric one. However, according to the data used by OPASKar and KRIMM [1] and KLABOE [3], the higher frequency band, which was observed at $1385 \mathrm{~cm}^{-1}$ in liquid 2 -chloropropane, has all of the characteristics of a symmetric, $A^{\prime}$, vibration: (a) its infrared gas phase spectrum has a strong central maximum with a weaker peak on each side of the central one [15]; (b) it is the more intense band in the Raman spectrum (the $1374 \mathrm{~cm}^{-1}$ band not even being observed); (c) it is polarized in the Raman spectrum. In order to more accurately calculate the relative frequencies of the $A^{\prime}$ and $A^{\prime \prime}$ umbrella vibrations in the $\mathrm{CH}_{3}$ groups, the diagonal force constant, $H_{\beta}$, for $\mathrm{C}-\mathrm{C}-\mathrm{H}$ bending, and the interaction force constant, $f_{\beta}$, between two such bendings in the same methyl group were allowed to vary. Although this violated the initial philosophy of our study, i.e. to maintain at a constant value all force constants which were transferred from the $n$-paraffin force field, we felt this to be justified because these constants were also found to predict inaccurately the relative values of the symmetric and antisymmetric umbrella modes in propane. GaYLES and Kivg [16] observed the infrared spectra of propane and its deuterium substituted derivatives and used the method of BADGER and ZUMWALT [15], and the GerharD-Drnnison [17] analysis of the asymmetric rotor, to assign the observed fundamental frequencies. In this study the $A_{1}$ umbrella vibration of propane was found to occur at

[13] A. V. R. WARRTER and S. Krimm, J. Chem. Phys. 52, 4316 (1970).

[14] R. G. SNYder, J. Chem. Phys. 47, 1316 (1967).

[15] R. M. Badger and L. R. ZuMwalt, J. Chem. Phys. 6, 711 (1938).

[16] J. N. GAYLES and W. T. KING, Spectrochim. Acta 21, 543 (1965).

[17] S. L. Gerhard and D. M. Dennison, Phys. Rev. 43, 197 (1933). 
$1391.9 \mathrm{~cm}^{-1}$ and the $B_{1}$ vibration at $1378 \mathrm{~cm}^{-1}$. With $H_{\beta}$ and $f_{\beta}$ unchanged, the $A_{1}$ mode in propane is calculated at $1370 \mathrm{~cm}^{-1}$ and the $B_{1}$ mode at $1382 \mathrm{~cm}^{-1}$ [14]. The new refined values of $H_{\beta}$ and $f_{\beta}$ predict the $A_{1}$ umbrella vibration in propane at $1386 \mathrm{~cm}^{-1}$ and the $B_{1}$ mode at $1372 \mathrm{~cm}^{-1}$. Similarly in 2-chloropropane with the new $H_{\beta}$ and $f_{\beta}$, the symmetric umbrella vibration observed at $1385 \mathrm{~cm}^{-1}$ is now calculated at $1386 \mathrm{~cm}^{-1}$, and the antisymmetric vibration, which is observed at $1374 \mathrm{~cm}^{-1}$ is now calculated at $1379 \mathrm{~cm}^{-1}$.

The existence of a specific intermolecular vibration in secondary chlorides which involves the $\mathrm{C}-\mathrm{Cl}$ bond, i.e. a $\mathrm{C}-\mathrm{Cl}$. . H-C interaction, has been strongly suggested by experimental evidence $[13,18,19]$. The force field reported here includes stretching and bending force constants to describe such an intermolecular interaction.

Prior to this work, the 2,4-dichloropentanes have been studied extensively $[1,5,20,21]$ because they are excellent model compounds of the poly(vinyl chloride) polymers. Under the assumption of tetrahedral geometry and staggered molecular conformation, each of the two stereoisomers of 2,4-dichloropentane can assume six unique rotational conformations. Theoretically each stereoisomer exists as a mixture of all six of its own rotational isomers. By investigating the $\mathrm{C}-\mathrm{Cl}$ stretching spectra of these molecules, SHrmaroucHI and TASUMI [2] concluded that the DL form of 2,4-dichloropentane existed mainly in the $T T$ form and that the meso form was so dominated by the $T G^{\prime}$ conformation that infrared spectra contained no observable absorption due to other conformations. We have found this not to be true. Attempts to explain the infrared, Raman, and NMR spectra of the 2,4-dichloropentanes on the basis of only the most prevalent structures have failed [5, 21]. In this study, observed bands in the far infrared at $150 \mathrm{~cm}^{-1}$ in DL- and at $160 \mathrm{~cm}^{-1}$ in $m e s o-2,4-$ dichloropentane could not be predicted by means of normal coordinate analyses of the preferred conformers. TASUMI et al. [4] were unable to predict bands in the vicinity of $180 \mathrm{~cm}^{-1}$ for PVC. These bands and their assignment to less prevalent conformations will be discussed in a subsequent publication.

The final force field was obtained by a refinement of 42 force constants to predict over 90 observed frequencies of chloropropane, ${ }_{\mathrm{H}} S_{\mathrm{H}}$ and ${ }_{\mathrm{H}} S_{\mathrm{O}}$ 2-chlorobutane, the ${ }_{\mathrm{H}} S_{\mathrm{H}}{ }_{\mathrm{H}} S_{\mathrm{H}}$ form of DL-2,4-dichloropentane, and the ${ }_{\mathrm{H}} S_{\mathrm{H}}{ }_{\mathrm{C}} S_{\mathrm{H}}$ form of meso-2,4dichloropentane. The final refined force field is given in Table 1.

\section{Model Compounds Used in Refinement Procedure}

\section{2-Chloropropane}

By using the theory of Badger and Zumwalt [15], Opaskar and Krimm [1] analyzed the symmetry of the absorption bands of the gas phase infrared spectrum of 2-chloropropane, and with the aid of normal coordinate analyses assigned these observed bands. The results of using the force field of Table 1 in a normal coordinate analysis of 2-chloropropane are given in Table 2. The assignments reported here agree with those of Ref [1]. The agreement between observed and calculated normal

[18] A. V. R. WARRIER and S. KRIMM, Macromolecules 3, 709 (1970).

[19] W. H. Moore and S. KrIMm, J.Am. Chem. Soc., in press.

[20] P. J. Flory and A. D. Williams, J. Am. Chem. Soc. 91, 3118 (1969).

[21] S. KRIMM, Pure Appl. Chem. 16, 369 (1968). 
Table 1. Force constants for secondary chlorides

\begin{tabular}{|c|c|c|c|}
\hline Name & Value§ & Environment & Coordinates coupled \\
\hline${ }^{*} K_{\mathrm{r}}$ & 4.6990 & $\mathrm{C}(\mathrm{H}, \mathbf{H}, \mathbf{H})$ & (C, $\mathrm{H})$ \\
\hline${ }^{*} K_{d}^{*}$ & 4.5380 & $\mathrm{C}(\mathbf{H}, \mathbf{H})$ & (C, H) \\
\hline$\dagger K_{d \mathrm{Ol}}$ & 4.8460 & $\mathrm{C}(\mathrm{H}, \mathrm{Cl})$ & $(\mathrm{C}, \mathrm{H})$ \\
\hline${ }^{*} K_{R}$ & 4.532 & $\mathrm{C}(\mathrm{H}, \mathrm{H})-\mathrm{C}(\mathrm{H}, \mathrm{H})$ & $(\mathrm{C}, \mathrm{C})$ \\
\hline$K_{\mathrm{RCl}}$ & 4.7047 & $\mathrm{C}(\mathrm{H}, \mathrm{H})-\mathrm{C}(\mathrm{H}, \mathrm{Cl})$ & $(C, C)$ \\
\hline$K_{\mathrm{CI}}$ & 2.7844 & $\mathrm{C}(\mathrm{H}, \mathrm{Cl})$ & $(\mathrm{C}, \mathrm{Cl})$ \\
\hline${ }^{*} H_{a}$ & 0.5390 & $\mathrm{C}\left(\mathrm{H}_{1}, \mathrm{H}_{2}, \mathrm{H}_{8}\right)$ & $\left(\mathrm{H}_{1}, \mathrm{C}, \mathrm{H}_{2}\right)$ \\
\hline$H_{\beta}^{\alpha}$ & 0.6213 & $\mathrm{C}\left(\mathrm{H}_{1}, \mathrm{H}_{2}, \mathrm{H}_{3}\right)-\mathrm{C}$ & (c) \\
\hline$*^{*} \tilde{H}_{\gamma}$ & 0.663 & & $\left(C_{1}, C_{2}, H_{1}\right)$ \\
\hline$H_{\varphi}$ & 0.6567 & $\mathrm{C}_{1}-\mathrm{C}_{2}(\mathrm{H}, \mathrm{Cl})$ & $\left(\mathrm{C}_{1}, \mathrm{C}_{2}, \mathrm{H}\right)$ \\
\hline$H_{\Xi}$ & 1.1062 & $\mathrm{G}_{1}-\mathrm{C}_{2}(\mathrm{H}, \mathrm{C} 1)$ & $\left(\mathrm{C}_{1}, \mathrm{C}_{8}, \mathrm{Cl}\right)$ \\
\hline$H_{1 \omega}$ & 0.9792 & $\mathrm{C}_{1}-\mathrm{C}_{2}(\mathrm{H}, \mathrm{Cl})-\mathrm{C}_{3}$ & \\
\hline$*_{2_{\omega}}$ & 1.032 & $\mathrm{C}_{1}^{*}-\mathrm{C}_{2}(\mathrm{H}, \mathrm{H})-\mathrm{C}_{3}$ & $\mathrm{C}^{3}$ \\
\hline$* H_{1 \delta}$ & 0.533 & $\mathrm{C}_{1}(\mathrm{H}, \mathrm{H})-\mathrm{C}_{2}\left(\mathrm{H}_{1}, \mathrm{H}_{2}\right)-\mathrm{C}_{3}(\mathrm{H}, \mathrm{H})$ &, $\left.\mathrm{H}_{2}\right)$ \\
\hline $\begin{array}{l}H_{2 \delta} \\
H_{3 \delta}\end{array}$ & $\begin{array}{l}0.5028 \\
0.5198\end{array}$ & $\begin{array}{l}\mathrm{C}_{1}(\mathrm{H}, \mathrm{Cl})-\mathrm{C}_{2}\left(\mathrm{H}_{1}, \mathrm{H}\right)-\mathrm{C}_{3}(\mathrm{H}, \mathrm{Cl}) \\
\mathrm{C}_{1}(\mathrm{H}, \mathrm{H})-\mathrm{C}_{2}\left(\mathrm{H}_{1}, \mathrm{H}_{2}\right)-\mathrm{C}_{3}(\mathrm{H}, \mathrm{Cl})\end{array}$ & $\left.\mathrm{H}_{2}\right)$ \\
\hline$H_{1 r}$ & 0.1105 & $\mathrm{C}_{1}(\mathrm{H}, \mathrm{H}, \mathrm{H})-\mathrm{C}_{2}(\mathrm{H}, \mathrm{Cl})-\mathrm{C}$ & \\
\hline$H_{\mathbf{2} \tau}$ & 0.0775 & $\mathrm{C}_{1}(\mathrm{H}, \mathrm{H}, \mathrm{H})-\mathrm{C}_{2}(\mathrm{H}, \mathrm{H})-\mathrm{C}$ & \\
\hline$H_{3_{\tau}}$ & 0.1309 & $\mathrm{C}_{1}(\mathrm{H}, \mathrm{H}, \mathrm{H})-\mathrm{C}_{2}\left(\mathrm{H}_{2}, \mathrm{Cl}\right)-\mathrm{C}(\mathrm{H}, \mathrm{H}, \mathrm{H})$ & \\
\hline$H_{\mathbf{4}_{\mathrm{T}}}$ & 0.0744 & $\mathrm{C}-\mathrm{C}_{1}(\mathrm{H}, \mathrm{H})-\mathrm{C}_{2}(\mathrm{H}, \mathrm{Cl})-\mathrm{C}$ & \\
\hline${ }^{*} F_{\text {, }}$ & 0.032 & $\mathrm{C}\left(\mathrm{H}_{1}, \mathrm{H}_{2}, \mathrm{H}_{3}\right)$ & $\left(\mathrm{C}, \mathrm{H}_{1}\right)\left(\mathrm{C}, \mathrm{H}_{2}\right)$ \\
\hline${ }^{*} F_{2 d}$ & 0.019 & $\mathrm{C}\left(\mathrm{H}_{1}, \mathrm{H}_{2}\right)$ & $\left(\mathrm{C}, \mathrm{H}_{1}\right)\left(\mathrm{C}, \mathrm{H}_{\mathbf{2}}\right)$ \\
\hline$*^{*} F_{1 R}$ & 0.083 & $\mathrm{C}_{1}(\mathrm{H}, \mathrm{H})-\mathrm{C}_{2}(\mathrm{H}, \mathrm{H})-\mathrm{C}_{3}(\mathrm{H}, \mathrm{H})$ & $\left(\mathrm{C}_{1}, \mathrm{C}_{8}\right)\left(\mathrm{C}_{2}, \mathrm{C}_{3}\right)$ \\
\hline$F_{2 R}$ & 0.177 & $\mathrm{C}_{1}(\mathrm{H}, \mathrm{Cl})-\mathrm{C}_{2}(\mathrm{H}, \mathrm{H})-\mathrm{C}_{3}(\mathrm{H})$ & $\left(\mathrm{C}_{1}, \mathrm{C}_{2}\right)\left(\mathrm{C}_{2}, \mathrm{C}_{3}\right)$ \\
\hline$F_{3 R}$ & 0.4058 & $\mathrm{C}_{1}(\mathrm{H}, \mathrm{H})-\mathrm{C}_{2}(\mathrm{H}, \mathrm{Cl})-\mathrm{C}_{3}(\mathrm{H}, \mathrm{H})$ & $\left(C_{1}, C_{2}\right)\left(C_{2}^{-}, C_{3}\right)$ \\
\hline$F_{R \mathrm{Cl}}$ & 0.6401 & $\mathrm{C}_{1}-\mathrm{C}_{2}(\mathrm{H}, \mathrm{Gl})$ & $\left(\mathrm{C}_{1}, \mathrm{C}_{2}\right)\left(\mathrm{C}_{2}, \mathrm{Cl}\right)$ \\
\hline${ }^{*} F_{1 R \gamma}$ & 0.174 & $\mathrm{C}_{1}-\mathrm{C}_{2}(\mathrm{H}, \mathrm{H})$ & $\left(\mathrm{C}_{1}, \mathrm{C}_{2}\right)\left(\mathrm{C}_{1}, \mathrm{C}_{2}, \mathrm{H}\right)$ \\
\hline$F_{2 R Y}^{\prime}$ & 0.1845 & $\mathrm{C}_{1}\left(\mathrm{H}_{1}, \mathrm{Cl}\right)-\mathrm{C}_{2}(\mathrm{H}, \mathrm{H})$ & $\left(\mathrm{C}_{1}^{\prime}, \mathrm{C}_{2}\right)\left(\mathrm{C}_{1}, \mathrm{C}_{2}, \mathrm{H}\right)$ \\
\hline$F_{3 B \gamma}$ & 0.1701 & $\mathrm{C}_{1}\left(\mathrm{H}_{1}, \mathrm{Cl}\right)-\mathrm{C}_{2}(\mathrm{H}, \mathrm{H})$ & $\left(\mathrm{C}_{1}, \mathrm{C}_{2}\right)\left(\mathrm{C}_{2}, \mathrm{C}_{1}, \mathrm{H}_{1}\right)$ \\
\hline$F_{1 R \omega}$ & 0.349 & $\mathrm{C}_{2}(\mathrm{H}, \mathrm{Cl})-\mathrm{C}_{2}-\mathrm{C}_{3}$ & $\left(C_{1}, C_{2}\right)\left(C_{1}, C_{2}, C_{3}\right) \ddagger$ \\
\hline${ }^{*} F_{2 R \omega}$ & 0.303 & $C_{2}-C_{2}-C_{3}$ & $\left(\mathrm{C}_{1}, \mathrm{C}_{2}\right)\left(\mathrm{C}_{2}^{-}, \mathrm{C}_{2}, \mathrm{C}_{8}\right)$ \\
\hline$F_{1 R \gamma}^{\prime \prime}$ & -0.0218 & $\mathrm{C}_{1}-\mathrm{C}_{2}\left(\mathrm{H}_{1}, \mathrm{H}_{2}\right)-\mathrm{C}_{3}(\mathrm{H}, \mathrm{Cl})$ & $\left(\mathrm{C}_{2}, \mathrm{C}_{8}\right)\left(\mathrm{C}_{1}, \mathrm{C}_{2}, \mathrm{H}_{1}\right)$ \\
\hline$F_{2 R \gamma}^{\prime}$ & -0.0868 & $\mathrm{C}_{1}-\mathrm{C}_{2}(\mathrm{H}, \mathrm{Cl})-\mathrm{C}_{3}$ & $\left(\mathrm{C}_{1}, \mathrm{C}_{2}\right)\left(\mathrm{C}_{3}, \mathrm{C}_{2}, \mathrm{H}\right)$ \\
\hline$*^{*} F_{B Y}$ & -0.097 & $\mathrm{C}_{1}^{2}-\mathrm{C}_{2}(\mathrm{H}, \mathrm{H})-\mathrm{C}_{3}$ & $\left(\mathrm{C}_{1}, \mathrm{C}_{2}\right)\left(\mathrm{C}_{3}, \mathrm{C}_{2}, \mathrm{H}\right)$ \\
\hline$F_{\omega \mathrm{Cl}}$ & -0.1117 & $\mathrm{C}_{1}-\mathrm{C}_{2}(\mathrm{H}, \mathrm{Cl})-\mathrm{C}_{3}^{\circ}$ & $\left(\mathrm{C}_{2}, \mathrm{C}\right)\left(\mathrm{C}_{1}, \mathrm{C}_{2}, \mathrm{C}_{8}\right)$ \\
\hline$F_{R} \Xi$ & 0.1003 & $\mathrm{C}_{1}-\mathrm{C}_{2}(\mathrm{H}, \mathrm{Cl})$ & $\left(\mathrm{C}_{1}^{-}, \mathrm{C}_{2}\right)\left(\mathrm{C}_{1}, \mathrm{C}_{2}, \mathrm{Cl}\right)$ \\
\hline$F_{\mathrm{Cl}}$ & 0.4005 & $\mathrm{C}_{1}-\mathrm{C}_{2}(\mathrm{H}, \mathrm{Cl})$ & $\left(\mathrm{C}_{2}, \mathrm{Cl}\right)\left(\mathrm{C}_{1}, \mathrm{C}_{2}, \mathrm{Cl}\right)$ \\
\hline$F_{\theta \mathrm{CI}}$ & 0.2399 & $\mathrm{C}(\mathrm{H}, \mathrm{Cl})$ & $(\mathrm{C}, \mathrm{Cl})(\mathrm{H}, \mathrm{C}, \mathrm{Cl})$ \\
\hline${ }^{*} F_{\beta \beta}$ & -0.0272 & $\mathbf{C}\left(\mathbf{H}_{1}, \mathbf{H}_{2}, \mathbf{H}_{3}\right)-\mathbf{C}$ & $\left(\mathrm{C}, \mathrm{C}, \mathrm{H}_{1}\right)\left(\mathrm{C}, \mathrm{C}, \mathrm{H}_{2}\right)$ \\
\hline${ }^{*} F_{\gamma \gamma}$ & -0.0190 & $\mathrm{C}\left(\mathrm{H}_{1}, \mathrm{H}_{2}\right)-\mathrm{C}$ & $\left(\mathrm{C}, \mathrm{C}, \mathrm{H}_{1}^{-}\right)\left(\mathrm{C}, \mathrm{C}, \mathrm{H}_{2}\right)$ \\
\hline${ }^{*} F_{y \gamma}^{\prime}$ & 0.0210 & $\mathrm{C}_{1}-\mathrm{C}_{2}\left(\mathrm{H}_{1}, \mathrm{H}_{2}\right)-\mathrm{C}_{3}$ & $\left(\mathrm{C}_{1}, \mathrm{C}_{2}, \mathrm{H}_{1}\right)\left(\mathrm{C}_{3}, \mathrm{C}_{2}, \mathrm{H}_{1}\right)$ \\
\hline${ }^{*} F_{1}^{\prime}{ }_{1 \gamma \omega}$ & -0.022 & $\mathrm{C}_{1}-\mathrm{C}_{2}\left(\mathrm{H}_{1}, \mathrm{H}_{2}^{-}\right)-\mathrm{C}_{3}$ & $\left(\mathrm{C}_{1}, \mathrm{C}_{2}, \mathrm{C}_{3}\right)\left(\mathrm{C}_{1}, \mathrm{C}_{2}, \mathrm{H}_{1}\right)$ \\
\hline$F_{2} \gamma_{\omega}$ & -0.0903 & $\mathrm{C}_{1}-\mathrm{C}_{2}(\mathrm{H}, \mathrm{Cl})-\mathrm{C}_{3}$ & $\left(\mathrm{C}_{2}, \mathrm{C}_{2}^{-}, \mathrm{C}_{3}\right)\left(\mathrm{C}_{1}^{-}, \mathrm{C}_{2}^{-}, \mathrm{H}\right)$ \\
\hline
\end{tabular}

* Force constants transferred from Ref. [7].

+ Force constant transferred from Ref. [1].

A chlorine atom is attached to at least one of the carbon atoms involved.

$\$$ Stretch constants are in units of $\mathrm{mdyn} / \AA$, stretch bend constants are in units of mdyn/rad, bend and torsion constants are in units of mdyn $\cdot \AA / \mathrm{rad}^{2}$.

U Superscripts $t, g$ imply that extreme atoms are trans and gauche to one another, respectively.

Trans implies that two angles are bisected by a common plane, gauche implies no such common bisector.

** op implies out-of-plane bending, ip implies in plane bending. 
Table 1. (continued)

\begin{tabular}{|c|c|c|c|}
\hline Name & Value§ & Environment & Coordinates coupled \\
\hline$F_{\varphi \theta}$ & 0.1055 & $\mathrm{C}_{1}-\mathrm{C}_{2}(\mathrm{H}, \mathrm{Cl})$ & $\left(\mathrm{C}_{1}, \mathrm{C}_{2}, \mathrm{H}\right)\left(\mathrm{H}, \mathrm{C}_{2}, \mathrm{Cl}\right)$ \\
\hline${ }^{*} f_{1 y y^{t}}$ & 0.073 & $\mathrm{C}_{1}\left(\mathrm{H}_{1}, \mathrm{H}_{2}\right)-\mathrm{C}_{8}\left(\mathrm{H}_{3}, \mathrm{H}_{4}\right)$ & $\left(\mathrm{H}_{1}, \mathrm{C}_{1}, \mathrm{C}_{2}\right)\left(\mathrm{C}_{1}, \mathrm{C}_{2}, \mathrm{H}_{3}\right) \|$ \\
\hline${ }^{*} f_{1} \gamma \gamma^{q}$ & -0.058 & $\mathrm{C}_{1}\left(\mathrm{H}_{1}^{2}, \mathrm{H}_{2}\right)-\mathrm{C}_{2}\left(\mathrm{H}_{3}, \mathrm{H}_{4}^{*}\right)$ & $\left(\mathrm{H}_{1}, \mathrm{C}_{1}, \mathrm{C}_{2}\right)\left(\mathrm{C}_{1}^{2}, \mathrm{C}_{2}, \mathrm{H}_{3}\right) \|$ \\
\hline${ }^{*} f_{1 \gamma \omega \omega^{g}}$ & -0.064 & $\mathrm{C}_{1}\left(\mathrm{H}_{1}, \mathrm{H}_{2}\right)-\mathrm{C}_{2}-\mathrm{C}_{3}$ & $\left(\mathrm{H}_{1}^{-}, \mathrm{C}_{1}^{-}, \mathrm{C}_{2}\right)\left(\mathrm{C}_{1}^{-}, \mathrm{C}_{2}, \mathrm{C}_{3}\right)$ \\
\hline${ }^{*} f_{1 \gamma \boldsymbol{\omega}}{ }^{t}$ & 0.073 & $\mathrm{C}_{1}\left(\mathrm{H}_{1}, \mathrm{H}_{2}\right)-\mathrm{C}_{2}-\mathrm{C}_{3}$ & $\left(\mathrm{H}_{1}, \mathrm{C}_{1}, \mathrm{C}_{2}\right)\left(\mathrm{C}_{1}, \mathrm{C}_{2}, \mathrm{C}_{3}\right)$ \\
\hline${ }^{*} f_{1} \omega \omega^{t}$ & 0.097 & $\mathrm{C}_{1}^{-}-\mathrm{C}_{2}-\mathrm{C}_{3}-\mathrm{C}_{4}$ & $\left(\mathrm{C}_{1}, \mathrm{C}_{8}, \mathrm{C}_{8}\right)\left(\mathrm{C}_{2}, \mathrm{C}_{3}, \mathrm{C}_{4}\right)$ \\
\hline${ }^{*} f_{1 \omega \omega \omega^{0}}$ & -0.005 & $C_{1}-C_{2}-C_{3}-C_{4}$ & $\left(\mathrm{C}_{1}, \mathrm{C}_{2}, \mathrm{C}_{3}\right)\left(\mathrm{C}_{2}, \mathrm{C}_{3}, \mathrm{C}_{4}\right)$ \\
\hline$f_{2 \omega \omega}^{t}$ & 0.095 & $\left.\mathrm{C}_{1}^{2}-\mathrm{C}_{2}(\mathrm{H}, \mathrm{C}]\right)-\mathrm{C}_{3}-\mathrm{C}_{4}$ & $\left(\mathrm{C}_{1}, \mathrm{C}_{2}, \mathrm{C}_{8}\right)\left(\mathrm{C}_{2}, \mathrm{C}_{3}, \mathrm{C}_{4}\right)$ \\
\hline$f_{3} \omega \omega^{g}$ & 0.0436 & $\mathrm{O}_{1}-\mathrm{C}_{2}(\mathrm{H}, \mathrm{Cl})-\mathrm{C}_{3}-\mathrm{C}_{4}$ & $\left(\mathrm{C}_{1}, \mathrm{C}_{2}, \mathrm{C}_{8}\right)\left(\mathrm{C}_{2}, \mathrm{C}_{3}, \mathrm{C}_{4}\right)$ \\
\hline$f_{2 \gamma \gamma}{ }^{t}$ & 0.0803 & $\mathrm{C}_{1}\left(\mathrm{H}_{1}, \mathrm{H}_{2}\right)-\mathrm{C}_{2}(\mathrm{H}, \mathrm{Cl})$ & $\left(\mathrm{H}_{1}, \mathrm{C}_{1}, \mathrm{C}_{2}\right)\left(\mathrm{C}_{1}, \mathrm{C}_{2}, \mathrm{H}\right)$ \\
\hline$f_{2} y \gamma^{g}$ & -0.0327 & $\mathrm{C}_{1}\left(\mathrm{H}_{1}, \mathrm{H}_{2}\right)-\mathrm{C}_{2}(\mathrm{H}, \mathrm{Cl})$ & $\left(\mathrm{H}_{1}, \mathrm{C}_{1}, \mathrm{C}_{2}\right)\left(\mathrm{C}_{1}, \mathrm{C}_{2}, \mathrm{H}\right)$ \\
\hline$f_{2} \gamma \omega^{t}$ & 0.073 & $\mathrm{C}_{1}(\mathrm{H}, \mathrm{Cl})-\mathrm{C}_{2}-\mathrm{C}_{3}$ & $\left(\mathrm{H}, \mathrm{C}_{1}, \mathrm{C}_{2}\right)\left(\mathrm{C}_{1}, \mathrm{C}_{2}, \mathrm{C}_{3}\right)$ \\
\hline$f_{2} \gamma \omega^{\circ}$ & -0.0077 & $\mathrm{C}_{1}(\mathrm{H}, \mathrm{Cl})-\mathrm{C}_{2}-\mathrm{C}_{3}$ & $\left(\mathrm{H}, \mathrm{C}_{1}, \mathrm{C}_{2}\right)\left(\mathrm{C}_{1}, \mathrm{C}_{2}, \mathrm{C}_{3}\right)$ \\
\hline$f_{y} \Xi^{g}$ & -0.1085 & $\mathrm{C}_{1}(\mathrm{H}, \mathrm{Cl})-\mathrm{C}_{2}\left(\mathrm{H}_{1}, \mathrm{H}_{2}\right)$ & $\left(\mathrm{Cl}, \mathrm{C}_{1}, \mathrm{C}_{2}\right)\left(\mathrm{C}_{1}, \mathrm{C}\right.$ \\
\hline$f_{\gamma} \Xi^{t}$ & -0.1548 & $\mathrm{C}_{1}^{2}(\mathrm{H}, \mathrm{Cl})-\mathrm{C}_{2}\left(\mathrm{H}_{1}, \mathrm{H}_{2}\right)$ & $\left(\mathrm{Cl}, \mathrm{C}_{1}, \mathrm{C}_{2}\right)\left(\mathrm{C}_{1}, \mathrm{C}_{2}, \mathrm{H}_{1}\right)$ \\
\hline$f_{\omega \Xi^{t}}$ & -0.0774 & $\mathrm{C}_{1}(\mathrm{H}, \mathrm{Cl})-\mathrm{C}_{2}-\mathrm{C}_{3}$ & $\left(\mathrm{Cl}, \mathrm{C}_{1}, \mathrm{C}_{2}\right)\left(\mathrm{C}_{1}^{-}, \mathrm{C}_{2}, \mathrm{C}_{3}\right)$ \\
\hline$f \omega \Xi^{g}$ & -0.0193 & $\mathrm{C}_{1}(\mathrm{H}, \mathrm{Cl})-\mathrm{C}_{2}^{2}-\mathrm{C}_{3}$ & $\left(\mathrm{Cl}, \mathrm{C}_{1}, \mathrm{C}_{\mathrm{g}}\right)\left(\mathrm{C}_{1}\right.$, \\
\hline$f_{y y^{\prime}}{ }^{t}$ & 0.0136 & $\mathrm{C}_{1}-\mathrm{C}_{2}\left(\mathrm{H}_{1}\right)-\mathrm{C}_{3}\left(\dot{\mathrm{H}}_{2}\right)$ & $\left(\mathrm{C}_{1}, \mathrm{C}_{2}, \mathrm{H}_{1}\right)\left(\mathrm{C}_{2}\right.$ \\
\hline$f_{y y}^{\prime \prime g}$ & 0.0519 & $\mathrm{C}_{1}-\mathrm{C}_{2}\left(\mathrm{H}_{1}\right)-\mathrm{C}_{3}\left(\mathrm{H}_{2}\right)$ & $\left(\mathrm{C}_{1}, \mathrm{C}_{2}, \mathrm{H}_{1}\right)\left(\mathrm{C}_{2}, \mathrm{C}\right.$ \\
\hline$f_{y y^{\prime \prime} t}$ & 0.0034 & $\mathrm{C}_{1}-\mathrm{C}_{2}\left(\mathrm{H}_{1}\right)-\mathrm{C}_{3}\left(\mathrm{H}_{2}\right)-\mathrm{C}_{4}$ & $\left(\mathrm{C}_{1}, \mathrm{C}_{2}, \mathrm{H}_{1}\right)\left(\mathrm{H}_{2}\right.$ \\
\hline$f_{y \gamma^{\prime \prime g}}^{\prime \prime g}$ & 0.0222 & $\mathrm{C}_{1}-\mathrm{C}_{2}\left(\mathrm{H}_{1}\right)-\mathrm{C}_{3}\left(\mathrm{H}_{2}\right)-\mathrm{C}_{4}$ & $\left.\left(\mathrm{C}_{1}, \mathrm{C}_{2}, \mathrm{H}_{1}\right) \mathrm{H}_{2}, \mathrm{C}_{3}, \mathrm{C}_{4}\right) \emptyset$ \\
\hline $\mathrm{K}_{\mathrm{Ol} \ldots \mathrm{H}}$ & 0.07 & $\mathrm{C}-\mathrm{Cl} \ldots \mathrm{H}-\mathrm{C}$ & $\mathrm{Cl} \ldots \mathrm{H}$ \\
\hline $\mathrm{H}_{\mathrm{a} \circ \mathrm{p}^{* *}}$ & 0.01 & $\mathrm{C}-\mathrm{Cl} \ldots \mathrm{H}-\mathrm{C}$ & $\mathrm{C}-\mathrm{CI} \ldots \mathrm{H}$ \\
\hline$H_{\alpha 1 p^{* *}}$ & 0.03 & $\mathrm{C}-\mathrm{Cl} \ldots \mathrm{H}-\mathrm{C}$ & $\mathrm{C}-\mathrm{Cl} \ldots \mathrm{H}$ \\
\hline$H_{\beta \circ p^{* *}}$ & 0.01 & $\mathrm{C}-\mathrm{Cl} \ldots \mathrm{H}-\mathrm{C}$ & $\mathrm{Cl} \ldots \mathrm{H}-\mathrm{C}$ \\
\hline$H_{\beta 1 \mathrm{p}}^{* *}$ & 0.25 & $\mathrm{C}-\mathrm{Cl} \ldots \mathrm{H}-\mathrm{C}$ & $\mathrm{Cl} \ldots \mathrm{H}-\mathrm{C}$ \\
\hline$f_{r \tau}$ & 0.008 & $\mathrm{C}_{1}-\mathrm{C}_{2}-\mathrm{C}_{3}-\mathrm{C}_{4}$ & \\
\hline
\end{tabular}

frequencies are better (especially in the region below $600 \mathrm{~cm}^{-1}$ ) than that previously obtained [1]. This improved agreement is due to the inclusion of torsional modes and to the actual refinement of the secondary chloride force field. For 2-chloropropane, as well as for the other secondary chlorides which were studied, the correlation between observed and calculated frequencies in the regions of methyl bending and rocking was inferior to that in other spectral regions. This suggests that the force constants which were transferred from the potential field for hydrocarbons could be refined to predict more accurately $\mathrm{CH}_{3}$ bending and rocking modes in secondary chlorides.

\section{2-Chlorobutane}

The dependence of the $\mathrm{C}-\mathrm{Cl}$ stretching frequencies on structure, and the use of this relationship to identify the presence of various molecular conformations, is exemplified in the spectral assignments for 2-chlorobutane [1]. Observations of the infrared spectra of 2-chlorobutane at room and at low temperatures indicate that the $607 \mathrm{~cm}^{-1}$ carbon-chlorine stretching frequency increases in intensity at low temperatures. The behavior of this frequency, which is uniquely associated with the ${ }_{\mathrm{H}} S_{\mathrm{H}}$ conformation of 2-chlorobutane, is assumed to be characteristic of all absorption bands associated with this rotational isomer. Therefore, following the logic for assignments used by Opaskar and Krimm, all observed bands whose intensities increase at low temperatures were assigned to the ${ }_{\mathrm{H}} \mathcal{S}_{\mathrm{H}}$ form of 2-chlorobutane. Table 3 gives the observed spectrum of 2-chlorobutane, 
Table 2. Observed and calculated frequencies (in $\mathrm{cm}^{-1}$ ), potential energy distributions and band assignments of 2-chloropropane

\begin{tabular}{|c|c|c|c|c|}
\hline \multicolumn{2}{|c|}{ Observed } & \multicolumn{2}{|c|}{ Calculated } & \multirow{2}{*}{$\begin{array}{l}\text { Potential energy } \\
\text { distribution } \S\end{array}$} \\
\hline$\overline{\text { Frequency* }}$ & Symmetry $\dagger$ & Symmetry & $\overline{\text { Frequency }}$ & \\
\hline $256 \mathrm{vw}$ & $s$ & $A^{\prime}$ & 256 & $\tau_{1}(97)$ \\
\hline $273 \mathrm{w}$ & $A$ & $A^{\prime \prime}$ & 273 & $\tau_{2}(98)$ \\
\hline $324 \mathrm{w}$ & $A$ & $A^{\prime \prime}$ & 327 & $x_{\pi}(95)$ \\
\hline $335 \mathrm{~m}$ & $S$ & $A^{\prime}$ & 337 & $W_{2}(84)$ \\
\hline $423 \mathrm{~m}$ & $S$ & $A^{\prime}$ & 421 & $W_{1}(77), B_{1}(12)$ \\
\hline $611 \mathrm{vs}$ & $s$ & $A^{\prime}$ & 611 & $\mathbf{X}(83)$ \\
\hline$(677 \mathrm{vw})$ & & $A^{\prime}$ & $2 \times 335=670$ & \\
\hline$(760 \mathrm{w})$ & & $A^{\prime}$ & $423+335=758$ & \\
\hline 885 & $s$ & $A^{\prime}$ & 881 & $R_{1}(73), B_{1}(19)$ \\
\hline $933 \mathrm{mw}$ & $A$ & $A^{\prime \prime}$ & 924 & $B_{4}(59), B_{8}(27)$ \\
\hline $965 ?$ & & $A^{\prime \prime}$ & 955 & $B_{8}(52), B_{4}(25)$ \\
\hline (1025 gh) & & $A^{\prime}$ & $811+423=1034$ & \\
\hline $1061 \mathrm{vg}$ & $S$ & $A^{\prime}$ & 1049 & $B_{2}(63), B_{1}(11)$ \\
\hline $1129 \mathrm{~m}$ & $A$ & $A^{\prime \prime}$ & 1122 & $R_{2}^{2}(53), H_{\pi}(12)$ \\
\hline $1160 \mathrm{~s}$ & $S$ & $A^{\prime}$ & 1156 & $B_{1}(45), R_{1}^{\pi}(21)$ \\
\hline (1223 vw) & & $A^{\prime}$ & $2 \times 611=1222$ & \\
\hline $1260 \mathrm{vs}$ & $S$ & $A^{\prime}$ & 1256 & $H_{\sigma}(88)$ \\
\hline$(1308 \mathrm{vw})$ & $S$ & $A^{\prime}$ & $885+423=1308$ & \\
\hline $1328 \mathrm{w}$ & $A$ & $A^{\prime \prime}$ & 1327 & $H_{n}(58), R_{2}(29)$ \\
\hline $1374 \mathrm{~s}$ & $A$ & $A^{\prime \prime}$ & 1379 & $U_{\mathrm{z}}^{n}(83), H_{\mathrm{z}}(11)$ \\
\hline $1385 \mathrm{~s}$ & $S$ & $A^{\prime}$ & 1386 & $U_{1}(85), R_{1}(6)$ \\
\hline (1446s) & & $A^{\prime}$ & 1459 & $A_{1}(69), A_{2}(21)$ \\
\hline & & $A^{\prime \prime}$ & 1459 & $A_{4}(66), A_{8}(26)$ \\
\hline to & & $A^{\prime \prime}$ & 1460 & $A_{3}(64), A_{4}(24)$ \\
\hline (1465s) & & $A^{\prime}$ & 1461 & $A_{2}(69), A_{1}(21)$ \\
\hline
\end{tabular}

* Liquid phase frequenoies.

$\dagger$ Symmetry from gas phase contours.

$\ddagger$ Refs. [1] and [9].

$\$$ For definitions of torsion coordinates see Ref. [9], for others see Ref. [1].

Frequencies in parentheses were not used in refinement procedure.

its behavior with respect to temperature, and the corresponding band assignments. The calculated potential energy distributions are reported in Table 4. The frequencies of the ${ }_{\mathrm{H}} \mathcal{S}_{\mathrm{H}^{\prime}}$ rotational isomer were not used in the refinement procedure.

Throughout this investigation, tetrahedral geometry and staggered conformations were assumed. These are popular, although arbitrary, assumptions. We know [22] that the $\mathrm{C}-\mathrm{C}-\mathrm{C}$ angle in secondary chlorides is closer to $112^{\circ}$ than to the $109^{\circ} 28^{\prime}$ associated with tetrahedral geometry. In the most recent refinement of the force field for $n$-paraffins [14], the value of the dihedral angle for gauche $\mathrm{C}-\mathrm{C}$ bonds was set at $\pm 67^{\circ}$. The discrepancy between the observed frequency $\left(627 \mathrm{~cm}^{-1}\right)$ of the $\mathrm{C}-\mathrm{Cl}$ stretching vibration in ${ }_{\mathrm{H}^{\prime}} S_{\mathrm{H}^{\prime}}$ 2-chlorobutane and the calculated value $\left(642 \mathrm{~cm}^{-1}\right)$ reflects the inaccuracies in the staggered approximation and in the tetrahedral configuration. To demonstrate the sensitivity of the carbon-chlorine stretching frequency to torsion angle in the ${ }_{\mathrm{H}} S_{\mathrm{H}^{\prime}}$ isomer of 2-chlorobutane, the $\mathrm{C}-\mathrm{Cl}$ stretching frequency is plotted in Fig. 1 as a function of $\tau$, the dihedral angle. This figure clearly shows that a $10^{\circ}$ change in torsion angle changes the $\mathrm{C}-\mathrm{Cl}$ stretching frequency in the ${ }_{\mathrm{H}} S_{\mathrm{H}^{\prime}}$ isomer by approximately $17 \mathrm{~cm}^{-1}$. At the same time the frequencies of $\mathrm{C}-\mathrm{Cl}$ stretching vibrations in the ${ }_{\mathrm{H}} S_{\mathrm{H}}$ and ${ }_{\mathrm{H}} S_{\mathrm{C}}$ isomers are relatively insensitive to variations of $\pm 10^{\circ}$ in the dihedral angle.

[22] F. L. Tobiason and R. H. Schwendeman, J. Chem. Phys. 40, 1014 (1964). 
Table 3. Assignments to observed frequencies (in $\mathrm{cm}^{-1}$ ) of 2-chlorobutane

\begin{tabular}{|c|c|c|c|}
\hline \multirow{2}{*}{$\begin{array}{l}\text { Observed liquid } \\
\text { frequency } \S\end{array}$} & \multicolumn{3}{|c|}{ Calculated frequency } \\
\hline & ${ }_{\mathbf{H}} S_{\mathbf{H}} \|$ & ${ }_{\mathbf{H}} S_{\mathrm{C}} \|$ & ${ }_{\mathrm{H}} S_{\mathrm{H}} \cdot \mathrm{T}$ \\
\hline \multirow[t]{2}{*}{77} & 77 & 83 & 72 \\
\hline & 189 & 200 & 195 \\
\hline $226^{*}$ & 223 & $(227)$ & 215 \\
\hline 244 & 245 & (250) & 243 \\
\hline $290 \mathrm{w}$ & & (308) & \\
\hline $324 \mathrm{~m}$ & 321 & & 334 \\
\hline $333 \mathrm{sh}$ & & & 341 \\
\hline $374 \mathrm{~m} \dagger$ & & 375 & \\
\hline $386 \mathrm{~m} *$ & 388 & & \\
\hline $418 \mathrm{mw} \dagger$ & & 423 & \\
\hline $460 \mathrm{mw}^{*}$ & 461 & & \\
\hline $522 \mathrm{~m} \dagger$ & & & 514 \\
\hline $607 \mathrm{vs}^{*}$ & 612 & & \\
\hline $627 \mathrm{~m} \dagger$ & & & 642 \\
\hline $670 \mathrm{st}$ & & 671 & \\
\hline $790 \mathrm{vs*}$ & 791 & 788 & 793 \\
\hline $822 \mathrm{~m} \dagger$ & & & 840 \\
\hline $843 \mathrm{~s}^{*}$ & 841 & 852 & \\
\hline $950 \mathrm{mst}$ & & 947 & 944 \\
\hline $958 \mathrm{~ms}^{*}$ & 957 & & \\
\hline $976 \mathrm{w \dagger}$ & & 970 & \\
\hline $992 \mathrm{~m}^{*}$ & 996 & & 991 \\
\hline $1000(?) \dagger$ & & & 997 \\
\hline $1010 \mathrm{wt}$ & & 1008 & \\
\hline $1022 w^{*}$ & 1016 & & \\
\hline $1060 \mathrm{~m} \dagger$ & & 1067 & 1064 \\
\hline $1073 \mathrm{mw}^{*}$ & 1071 & & \\
\hline $1108 \mathrm{w}^{*}$ & 1106 & & \\
\hline $1118 \mathrm{~m} \dagger$ & & & 1115 \\
\hline $1140 \mathrm{vw}$ & & $(1135)$ & 1145 \\
\hline $1157 \mathrm{st}$ & 1162 & (1159) & \\
\hline $1238 \mathrm{vs} \ddagger$ & 1231 & $(1245)$ & 1236 \\
\hline $1265 \mathrm{vw}$ & & $(1255)$ & \\
\hline $1286 \mathrm{~s}^{*}$ & 1291 & & 1280 \\
\hline $1297 \mathrm{~s}^{*}$ & 1302 & & \\
\hline $1320 \mathrm{mw} \dagger$ & & $(1318)$ & 1322 \\
\hline $1347 \mathrm{vw}$ & & & 1351 \\
\hline $1360 w^{*}$ & 1353 & $(1362)$ & \\
\hline 1382 vst & 1384 & $(1381)$ & 1381 \\
\hline & 1395 & 1398 & 1393 \\
\hline $1430 \mathrm{vw}$ & (1426) & $(1428)$ & 1428 \\
\hline 1447 vst & (1458) & $(1458)$ & 1458 \\
\hline & (1458) & $(1458)$ & 1459 \\
\hline & (1460) & $(1460)$ & 1460 \\
\hline $1460 \mathrm{vs} \ddagger$ & (1462) & (1462) & 1462 \\
\hline
\end{tabular}

* Intensity increases at low temperatures.

$\uparrow$ Intensity decreases at low temperatures.

$\ddagger$ Intensity remains strong at low temperulures.

$\$$ Ref. [1] and [9].

Frequencies in parentheses were not assigned before refinement.

If The ${ }_{\mathrm{H}} S_{\mathrm{H}} \cdot$ isomer was not used in refinement.

Besides the assignments of the far infrared absorption spectra of 2-chlorobutane, the assignments of the observed bands at 1297 and $1286 \mathrm{~cm}^{-1}$ differ from the assignments of OPASKaR and KrIMM [1]. These bands, which were both reported not to change in relative intensity upon cooling, were each assigned to both the ${ }_{H} S_{H}$ and ${ }_{H} S_{C}$ isomers. We have assigned the band at $1286 \mathrm{~cm}^{-1}$ to the ${ }_{\mathrm{H}} S_{\mathrm{H}}$ and ${ }_{\mathrm{H}} S_{\mathrm{H}^{\prime}}$ isomers and the $1297 \mathrm{~cm}^{-1}$ band only to the ${ }_{\mathrm{H}} S_{\mathrm{H}}$ conformation. Our assignments were made 
Table 4. Calculated frequencies (in $\mathrm{cm}^{-1}$ ) and potential energy distributions for 2-chlorobutane

Frequency

(a) $\mathrm{H}_{\mathrm{H}}$ conformation Potential energy distribution*

$\begin{array}{rl}77 & \tau_{2}(92) \\ 189 & \tau_{3}(83) \\ 223 & C C C(39), W_{2}(24) \\ 245 & \tau_{1}(91) \\ 321 & W_{2}(49), X_{z}(41) \\ 388 & W_{1}(52), C C C(16) \\ 461 & C C C(27), W_{1}(24) \\ 612 & X(80), W_{2}(8) \\ 791 & r(55), B_{2 l}(42) \\ 841 & R_{2}(39), B_{1 t}(21) \\ 957 & B_{2 r}(43), H_{2}(16) \\ 996 & B_{1 r}(24), w(16) \\ 1016 & R_{3}(30), R_{1}(20) \\ 1071 & B_{2 r}(17), r(15) \\ 1106 & R_{1}(43), R_{2}(10) \\ 1162 & R_{2}(23), B_{1 r}(17) \\ 1231 & H_{\sigma}(53), t(19) \\ 1291 & H_{\sigma}(27), t(26) \\ 1302 & H_{\pi}(28), w(22) \\ 1353 & U_{l}(23), U_{r}(21) \\ 1384 & U_{r}(56), U_{l}(33) \\ 1395 & U_{l}(31), w(22) \\ 1426 & \delta(88) \\ 1458 & A_{1 r}(51), A_{1 l}(32) \\ 1458 & A_{2 l}(88) \\ 1460 & A_{2 r}(81), A_{1 r}(8) \\ 1462 & A_{1 l}(54), A_{1 r}(29) \\ & \end{array}$

(b) ${ }_{\mathrm{H}} \mathrm{S}_{\mathrm{C}}$ conformation

$\begin{array}{rl}83 & \tau_{2}(80), \tau_{3}(18) \\ 200 & \tau_{8}(78) \\ 227 & W_{2}(31) \\ 250 & \tau_{1}(69), W_{2}(14) \\ 308 & X_{r}(79) \\ 375 & C C C(29), X(25) \\ 423 & W_{1}(67), W_{2}(14) \\ 671 & X(82), W_{2}(15) \\ 788 & r(53), B_{22}(39) \\ 852 & R_{2}(39), B_{1 l}(16) \\ 947 & B_{2 r}(28), B_{1 l}(17) \\ 970 & t(23), B_{2 l}(22) \\ 1008 & R_{3}(27), B_{2 r}(24) \\ 1067 & B_{1 l}(27), R_{3}(21) \\ 1135 & R_{1}(27), R_{2}(14) \\ 1159 & B_{1 r}(18), R_{2}(17) \\ 1245 & H_{\sigma}(70), t(7) \\ 1255 & t(34), H_{\sigma}(20) \\ 1318 & w(28), t(22) \\ 1362 & U_{r}(31), H_{r}(15) \\ 1381 & U_{l}(45), U_{r}(42) \\ 1398 & U_{l}(24), w(23) \\ 1428 & \delta(83) \\ 1458 & A_{2 l}(34), A_{1 r}(33) \\ 1458 & A_{2 l}(57), A_{1 r}(21) \\ 1460 & A_{2 r}(86) \\ 1462 & A_{12}(54), A_{1 r}(29) \\ & \end{array}$

* For definitions of torsion coordinates see Ref. [9], for others see Ref. [1].

$\dagger$ The ${ }_{H} S_{H^{\prime}}$ conformation was not used in the refinement procedures. 
Table 4. (continued)

\begin{tabular}{|c|c|}
\hline Frequency & Potential energy distribution* \\
\hline \multicolumn{2}{|c|}{ (c) ${ }_{\mathrm{H}} S_{\mathrm{H}^{\prime}} \uparrow$ conformation } \\
\hline 72 & $\tau_{2}(93)$ \\
\hline 195 & $\tau_{3}(87)$ \\
\hline 215 & $\operatorname{CCC}(49), W_{1}(31)$ \\
\hline 243 & $\tau_{1}(93)$ \\
\hline $\mathbf{3 3 4}$ & $X_{z}(52), W_{2}(27)$ \\
\hline 341 & $W_{2}(52), X_{\pi}(26)$ \\
\hline 514 & $X(33), \operatorname{CCC}(25)$ \\
\hline 642 & $X(71), W_{1}(22)$ \\
\hline 793 & $r(53), B_{2 l}(44)$ \\
\hline 840 & $R_{2}(45), R_{3}(16)$ \\
\hline 944 & $R_{3}(24), R_{1}(22)$ \\
\hline 991 & $B_{2 r}(30), B_{1 r}(21)$ \\
\hline 997 & $B_{1 l}(19), B_{1 r}(17)$ \\
\hline 1064 & $B_{1 l}(18), B_{27}(15)$ \\
\hline 1115 & $B_{1 \tau}(19), R_{\mathrm{g}}(11)$ \\
\hline 1145 & $R_{1}(29), r(14)$ \\
\hline 1230 & $H_{\sigma}(58), t(17)$ \\
\hline 1280 & $t(39), H_{\sigma}(23)$ \\
\hline 1322 & $H_{\pi}(32), R_{2}(23)$ \\
\hline 1351 & $U_{l}^{\pi}(30), w(30)$ \\
\hline 1381 & $U_{r}(87)$ \\
\hline 1393 & $U_{l}(57), w(24)$ \\
\hline 1428 & $\delta(92)$ \\
\hline 1458 & $A_{2 l}(88)$ \\
\hline 1459 & $A_{17}(00)$ \\
\hline 1460 & $A_{2 r}(87)$ \\
\hline 1462 & $A_{12}(60), A_{1 r}(26)$ \\
\hline
\end{tabular}

solely on the basis of the results of the 2-chlorobutane normal coordinate analyses and the force field refinement. However, a very recent investigation by BENEDETTI and CECCHI [12] lends support to this assignment. Benedetti and Cecchi observed both the liquid and crystalline phase spectra of 2-chlorobutane. These spectra show that upon cooling the bands at 1297 and $1286 \mathrm{~cm}^{-1}$ increase in intensity. The intensity enhancement is greater at 1297 than at $1286 \mathrm{~cm}^{-1}$. This temperature dependence suggests two things: (1) not all of the isomers present in liquid 2-chlorobutane contribute to the absorptions at 1286 and $1297 \mathrm{~cm}^{-1}$; (2) the combination of isomers

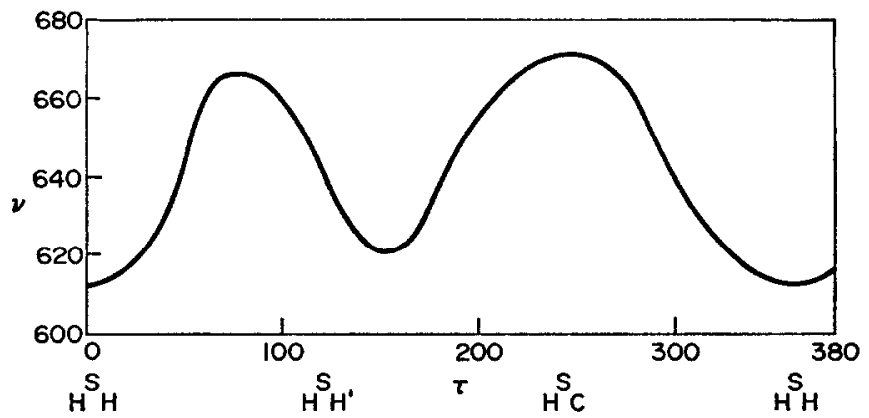

Fig. 1. Carbon -chlorino stretching frequency as a function of $\mathrm{C}_{2}-\mathrm{C}_{3}$ torsion angle in 2-chlorobutane. 
which contribute to the band at $1286 \mathrm{~cm}^{-1}$ is different from that which contributes to the $1297 \mathrm{~cm}^{-1}$ band. With the assignment of the absorption at $1286 \mathrm{~cm}^{-1}$ to both the ${ }_{\mathrm{H}} S_{\mathrm{H}}$ and ${ }_{\mathrm{H}} S_{\mathrm{H}^{\prime}}$ isomers, the intensity of this band would be expected to increase less at low temperatures than that of the $1297 \mathrm{~cm}^{-1}$ band, which at room temperatures has contributions only from the ${ }_{\mathrm{H}} S_{\mathrm{H}}$ isomeric form.

\section{2,4-Dichloropentane}

The ${ }_{\mathrm{H}} S_{\mathrm{H}}{ }_{\mathrm{H}} S_{\mathrm{H}}$ form of syndiotactic DL-2,4-dichloropentane and the ${ }_{\mathrm{H}} S_{\mathrm{H}} \mathrm{C}_{\mathrm{H}}$ form of isotactic meso-2,4-dichloropentane were also included in the least squares refinement. Results of normal coordinate calculations, and observed frequencies, are given in Tables 5 and 6.

Table 5. Observed and calculated frequencies (in $\mathrm{cm}^{-1}$ ) and potential energy distributions of $T T$ conformer of DL-2,4dichloropentane

\begin{tabular}{|c|c|c|}
\hline $\begin{array}{l}\text { Observed } \\
\text { frequency }\end{array}$ & $\begin{array}{l}\text { Caloulated } \\
\text { frequency }\end{array}$ & $\begin{array}{l}\text { Potential energy } \\
\text { distribution }\end{array}$ \\
\hline & $\begin{array}{l}42 A \\
58 B\end{array}$ & $\begin{array}{l}\tau_{A 2}(96) \\
\tau_{B 2}(90)\end{array}$ \\
\hline \multirow[t]{2}{*}{120} & $120 A$ & $\operatorname{CCC}(52), W_{\Delta 1}(29)$ \\
\hline & $240 \mathrm{~A}$ & $\tau_{A 1}(98)$ \\
\hline $245 \mathrm{vw}$ & $241 B$ & $\tau_{B 1}(91)$ \\
\hline 273 & $267 A$ & $W_{A 1}(43), X_{\pi A}(39)$ \\
\hline 310 & $312 B$ & $X_{\pi B}(44), W_{B 1}(33)$ \\
\hline $343 \mathrm{~m}$ & $342 \mathrm{~B}$ & $X_{R B}(42), W_{B 1}(39)$ \\
\hline $368 \mathrm{~m}$ & $371 A$ & $W_{A 2}(65), W_{A 1}(10)$ \\
\hline $460 \mathrm{~m}$ & $458 B$ & $W_{B 2}(74), W_{B 1}(12)$ \\
\hline$(478 w)$ & $472 A$ & $X_{\pi \Delta}(28), \operatorname{CCC}(26)$ \\
\hline $606 \mathrm{~s}$ & $603 B$ & $X_{B}(84)$ \\
\hline \multirow[t]{2}{*}{6278} & $625 \mathrm{~A}$ & $X_{A}(81)$ \\
\hline & $\{872 B$ & $r(59), B_{B 1}(23)$ \\
\hline$(877 \mathrm{~ms})$ & $\{884 A$ & $R_{A 2}(40), B_{A 1}(29)$ \\
\hline $938 \mathrm{~s}$ & $939 B$ & $R_{B 1}(25), R_{B 2}(23)$ \\
\hline $978 \mathrm{~m}$ & $985 A$ & $B_{A 2}(35), B_{d 1}(33)$ \\
\hline $1012 \mathrm{~s}$ & $1023 B$ & $B_{B 2}(61), R_{B 1}(10)$ \\
\hline $1067 \mathrm{~m}$ & $1068 B$ & $R_{B 1}(44), B_{B 1}(30)$ \\
\hline $1100 \mathrm{vw}$ & $1102 A$ & $R_{\Delta 1}(54), t(10)$ \\
\hline $1125 \mathrm{~s}$ & $1119 \mathrm{~A}$ & $R_{\Delta 2}(21), B_{A 2}(19)$ \\
\hline $1132 \mathrm{~s}$ & $1139 B$ & $R_{B 2}(42), H_{\pi B}(10)$ \\
\hline $1191 \mathrm{~m}$ & $1188 \mathrm{~A}$ & $t(28), H_{\sigma A}(23)$ \\
\hline $1222 \mathrm{vvw}$ & $2 \times 606=1212$ & \\
\hline $1257 \mathrm{~s}$ & $\begin{array}{l}1252 B \\
1280 B\end{array}$ & $\begin{array}{l}H_{\sigma B}(85) \\
w(44), H_{\pi B}(36)\end{array}$ \\
\hline \multirow{2}{*}{$\begin{array}{l}(1288 \mathrm{~s}) \\
1325 \mathrm{mw}\end{array}$} & $1292 A$ & $H_{\sigma A}(51), t(25)$ \\
\hline & $\begin{array}{l}1325 A \\
1361 B\end{array}$ & $\begin{array}{l}H_{z A}(56), R_{A 1}(15) \\
U_{B}(48), w(23)\end{array}$ \\
\hline \multirow{2}{*}{$1379 \mathrm{~s}$} & $1381 A$ & $U_{A}(89)$ \\
\hline & \multicolumn{2}{|c|}{ (1415 ms) } \\
\hline & $1427 A$ & $\delta(95)$ \\
\hline \multirow{4}{*}{$\begin{array}{l}(1443 \mathrm{~s}) \\
(1450 \mathrm{~m})\end{array}$} & $1459 \mathrm{~A}$ & $A_{A 1}(72), A_{A 2}(18)$ \\
\hline & $1459 B$ & $A_{B 1}(78), A_{B 2}(20)$ \\
\hline & $1459 B$ & $A_{B 2}(80), A_{B 1}(10)$ \\
\hline & $1460 A$ & $A_{A 2}(72), A_{A 1}(18)$ \\
\hline
\end{tabular}

* Ref. $[1]$ and [9].

+ For definitions of symmetry coordinates see Ref. [1] and [9]. Frequencies in parenthesis were not used in the refinement procedure. 
Table 6. Observed and calculated frequencies (in $\mathrm{cm}^{-1}$ ) and potential energy distributions of $T G^{\prime}$ conformer of meso-2,4. dichloropentane

\begin{tabular}{|c|c|c|}
\hline $\begin{array}{l}\text { Observed } \\
\text { frequency }\end{array}$ & $\begin{array}{l}\text { Calculated } \\
\text { frequency }\end{array}$ & $\begin{array}{l}\text { Potential energy } \\
\text { distribution" }\end{array}$ \\
\hline & 49 & $\tau_{B 2}(82), \tau_{A 2}(12)$ \\
\hline & 56 & $\tau_{A 2}(75), \tau_{B 2}(13)$ \\
\hline 118 & 129 & $\operatorname{CCC}(49), W_{B 1}(37)$ \\
\hline \multirow[t]{2}{*}{228} & 232 & $X_{\pi A}(54), \tau_{B 2}(22)$ \\
\hline & 242 & $\tau_{\Delta 1}(96)$ \\
\hline $245 w$ & 245 & $\tau_{B 1}(73)$ \\
\hline $315 \mathrm{~m}$ & 316 & $W_{A 1}(74)$ \\
\hline $345 \mathrm{w}$ & 342 & $X_{\# B}(51), W_{B 1}(23)$ \\
\hline $392 \mathrm{w}$ & 393 & $W_{A 2}(64), X_{\pi B}(8)$ \\
\hline $410 \mathrm{~ms}$ & 405 & $X_{\pi A}(18), \operatorname{CCC}(15)$ \\
\hline $460 \mathrm{w}$ & 466 & $W_{B 2}(63), W_{B 1}(9)$ \\
\hline $611 \mathrm{vs}$ & 615 & $X_{A}(61), X_{B}(42)$ \\
\hline $680 \mathrm{vs}$ & 674 & $X_{B}(45), X_{A}(37)$ \\
\hline $855 \mathrm{~s}$ & 861 & $r(42), R_{A 1}(10)$ \\
\hline $882 \mathrm{~m}$ & 895 & $R_{A 2}(25), B_{A 1}(21)$ \\
\hline $926 \mathrm{~s}$ & 921 & $B_{B 1}(26), R_{B 2}(22)$ \\
\hline $980 \mathrm{~ms}$ & 989 & $B_{B 1}(31), B_{A 2}(26)$ \\
\hline $1006 \mathrm{~s}$ & 1009 & $B_{B 2}(40), B_{A 2}(16)$ \\
\hline $1058 \mathrm{~s}$ & 1059 & $R_{A 1}(32), t(13)$ \\
\hline $1089 \mathrm{mw}$ & 1096 & $R_{B 1}(27), R_{A 1}(15)$ \\
\hline \multirow[t]{2}{*}{$1130 \mathrm{vs}$} & 1132 & $R_{B 1}(18), R_{A 2}(16)$ \\
\hline & 1145 & $R_{B 2}(22), B_{B 2}(12)$ \\
\hline $1199 \mathrm{~m}$ & 1196 & $t(31), H_{\sigma A}(26)$ \\
\hline $1237 \mathrm{~s}$ & 1246 & $H_{\sigma B}(50), H_{\sigma \Delta}(35)$ \\
\hline $1272 \mathrm{~s}$ & 1271 & $H_{\sigma B}(30), H_{\sigma A}(28)$ \\
\hline $1289 \mathrm{~m}$ & 1291 & $u(38), H_{\sigma A}(28)$ \\
\hline $1337 \mathrm{mw}$ & 1335 & $H_{\pi A}(40), t(22)$ \\
\hline 1360 & 1365 & $U_{B}(54), R_{B}(17)$ \\
\hline $1378 \mathrm{vs}$ & 1382 & $U_{A}(88)$ \\
\hline $1415 \mathrm{vw}$ & ?1398 & $U_{B}(39), H_{z B}(22)$ \\
\hline $1427 \mathrm{w}$ & 1429 & $\delta(91)$ \\
\hline \multirow{2}{*}{$1439 \mathrm{~s}$} & 1459 & $A_{A 1}(73), A_{B 2}(16)$ \\
\hline & 1460 & $A_{B 1}(69), A_{B 2}(16)$ \\
\hline \multirow[t]{2}{*}{$1453 \mathrm{~s}$} & 1460 & $A_{A 2}(59), A_{B 2}(15)$ \\
\hline & 1460 & $A_{B 2}(42), A_{A 2}(29)$ \\
\hline
\end{tabular}

* For definitions of symmetry coordinates see Ref. [1] and [9].

The force field for secondary chlorides predicts the observed spectra of the two dominant forms of $\mathrm{DL}$ - and meso-2,4-dichloropentane very well. In both isomers a band at $1415 \mathrm{~cm}^{-1}$ was observed. This absorption is strong in the spectrum of syndiotactic 2,4-dichloropentane and weak in the spectrum of the isotactic configuration. Absorption at $1415 \mathrm{~cm}^{-1}$ is also observed in the spectrum of isotactic 2,4,6-trichloroheptane [6]. The assignment of this band to a combined $\mathrm{H}-\mathrm{C}-\mathrm{Cl}$ bending and methyl bending is tentative. A Raman band at $1415 \mathrm{~cm}^{-1}$ in the spectrum of crystalline polyethylene has also caused assignment problems. This band was originally assigned to the $B_{2 g}$ methylene wagging mode [23]. More recently [14, 24] the wagging fundamental has been reassigned to an infrared band at $1382 \mathrm{~cm}^{-1}$ leaving

[23] J. H. Schachtschneider and R. G. SNyder, Spectrochim. Acta 19, 117 (1963).

[24] R. G. SNYDER, J. Mol. Spectry 23, 224 (1967). 
the Raman band at $1415 \mathrm{~cm}^{-1}$ unassigned. In 2,4-dichloropentane, 2,4,6-trichloroheptane, and in poly(vinyl chloride), bands appear at approximately $1427 \mathrm{~cm}^{-1}$. Strong absorption between 1447 and $1460 \mathrm{~cm}^{-1}$ is present in the spectra of all secondary chlorides. We assign this strong unresolved absorption to asymmetric methyl bending modes which occur in the same general region in the $n$-paraffins. The bands in the region around $1430 \mathrm{~cm}^{-1}$ have been assigned to the $\mathrm{HCH}$ scissors fundamental of the methylene group. This assignment is verified by observing the spectra of poly(vinyl chloride), in which effects of methyl bending are not present [25].

\section{Model Compounds Not Used for Forde Finld Refinement}

To establish the validity of the force field, it was used to calculate the normal frequencies of 3-chloropentane, axial and equatorial monochlorocyclohexane, and several rotational isomers of each of the stereochemical forms of 2,4,6-trichloroheptane. Detailed assignments of the observed absorption spectra of the stereoisomers of 2,4,6-trichloroheptane will be discussed in a subsequent publication.

The results of normal coordinate calculations of equatorial and axial monochlorocyclohexane parallel those previously reported by OPASKAR and KRIMM [1]. The ${ }_{\mathrm{C}} S_{\mathrm{C}}$ carbon-chlorine stretching frequenoy at $728 \mathrm{~cm}^{-1}$ in the equatorial form is calculated at $722 \mathrm{~cm}^{-1}$. The two observed absorptions at $557 \mathrm{~cm}^{-1}$ and at $683 \mathrm{~cm}^{-1}$ in axial monochlorocyclohexane are both $\mathrm{C}-\mathrm{Cl}$ stretching bands. This splitting of ${ }_{\mathrm{H}^{\prime}} \mathrm{S}_{\mathrm{H}^{\prime}} \mathrm{C}-\mathrm{Cl}$ stretching was previously predicted [1]. Although we calculate a splitting, viz. $543 \mathrm{~cm}^{-1}$ and $705 \mathrm{~cm}^{-1}$, the higher frequency does not agree well with the observed band at $683 \mathrm{~cm}^{-1}$. The $\mathrm{CH}_{2}-\mathrm{CHCl}-\mathrm{CH}_{2}$ environment was not present in any of the model compounds used in the refinement process. As a result, the force field was not refined to $a_{\mathrm{H}^{\prime}} \mathrm{S}_{\mathrm{H}^{\prime}} \mathrm{C}-\mathrm{Cl}$ stretching frequency. Moreover, in 2-chlorobutane, the frequency of the ${ }_{\mathrm{H}} S_{\mathrm{H}}{ }^{\prime} \mathrm{C}-\mathrm{Cl}$ stretching vibration was found to be very sensitive to small variations in the dihedral angle around the gauche $\mathrm{C}-\mathrm{C}$ bond. The discrepancy between the observed $\left(683 \mathrm{~cm}^{-1}\right)$ and calculated $\left(705 \mathrm{~cm}^{-1}\right)$ frequencies could presumably result from both of these factors.

\section{3-Chloropentane}

At room temperature, 3-chloropentane is considered to exist as a mixture of four rotational isomers, i.e. ${ }_{\mathrm{H}} S_{\mathrm{H}},{ }_{\mathrm{C}} S_{\mathrm{H}},{ }_{\mathrm{H}} S_{\mathrm{H}^{\prime}}$, and ${ }_{\mathrm{C}} S_{\mathrm{H}^{\prime}}$. The results of our calculations are given in Tables 7 and 8.

ShIPMaN et al. [10] and CaracUlacu [26] independently studied the isomeric composition of 3-chloropentane by correlating the observed spectra in the region of the $\mathrm{C}-\mathrm{Cl}$ stretching vibrations, $590-750 \mathrm{~cm}^{-1}$, with the rotational isomers believed to be characteristic of them. The study by Caraculacu included complete infrared and Raman spectra for liquid and solid phases of 3-chloropentane. In the region of the $\mathrm{C}-\mathrm{Cl}$ stretching vibrations, a band observed at $606 \mathrm{~cm}^{-1}$, which is assignable only to ${ }_{\mathrm{H}} S_{\mathrm{H}} \mathrm{C}-\mathrm{Cl}$ stretching, persists in the solid state Raman and infrared spectra. As in 2-chlorobutane, the behavior of this band was assumed to be characteristic of

[25] S. Krimm, V. L. Folt, and J. J. Shipman, J. Polymer Sci. A1, 2621 (1963).

[26] A. Caradulacu, J. Śtokr and B. Schnemer, Collection Czech. Chem. Commun. 29, 2783 (1964). 
Table 7. Observed and calculated frequencies (in $\mathrm{cm}^{-1}$ ) of 3-chloropentane

\begin{tabular}{|c|c|c|c|c|c|c|c|}
\hline \multicolumn{4}{|c|}{ Observed frequency* } & \multirow{2}{*}{\multicolumn{4}{|c|}{ Calculated frequency }} \\
\hline \multicolumn{2}{|c|}{ Infrared } & \multicolumn{2}{|c|}{ Raman } & \multirow[b]{2}{*}{${ }_{\mathrm{H}} S_{\mathrm{H}}$} & \multirow[b]{2}{*}{$\alpha S_{\mathbf{H}}$} & & \\
\hline Liquid & Solid & Liquid & Solid & & & $\mathbf{H} S_{\mathbf{H}}$. & $\mathbf{c} S_{\mathrm{H}}$ \\
\hline & & & & & 72 & 69 & 55 \\
\hline & & 72 & 72 & 66 & & & \\
\hline & & 80 & 80 & 82 & 78 & 74 & 91 \\
\hline & & 168 & 164 & 174 & 180 & 173 & 185 \\
\hline & & 194 & & 197 & 201 & 194 & 193 \\
\hline & & 206 & 200 & 202 & & 201 & \\
\hline & & & & & 226 & & \\
\hline & & 238 & 241 & 258 & 243 & & $\begin{array}{l}236 \\
241\end{array}$ \\
\hline & & 292 & - & & & 274 & \\
\hline & & & & & & 331 & 320 \\
\hline & & 362 & - & & $\mathbf{3 4 8}$ & & \\
\hline & & $374 \mathrm{~ms}$ & $371 \mathrm{~ms}$ & 362 & & & \\
\hline 400 & - & $398 \mathrm{~s}$ & - & & 397 & 408 & 381 \\
\hline 425 vw & $425 \mathrm{w}$ & $426 \mathrm{~s}$ & 427 B & 430 & & & \\
\hline 460 vw & - & $457 w$ & - & 474 & 464 & & \\
\hline $533 \mathrm{~m}$ & - & $533 \mathrm{~ms}$ & - & & & 541 & 530 \\
\hline $606 \mathrm{~m}$ & $594 \mathrm{~B}$ & 606 a & $594 \mathrm{vg}$ & 615 & & & \\
\hline $634 \mathrm{~m}$ & - & $633 \mathrm{~ms}$ & - & & & 641 & \\
\hline $657 \mathrm{sh}$ & - & $657 \mathrm{~s}$ & - & & 675 & & \\
\hline $667 \mathrm{sh}$ & - & $668 \mathrm{sh}$ & - & & & & 694 \\
\hline $776 \mathrm{~m}$ & $768 \mathrm{vw}$ & $775 \mathrm{vw}$ & 762 & 774 & 777 & & 785 \\
\hline $795 \mathrm{~m}$ & - & $793 \mathrm{vw}$ & 一 & & & 783 & 800 \\
\hline $811_{8}$ & - & $812 \mathrm{~ms}$ & - & & 804 & 806 & \\
\hline 826 B & $820 \mathrm{~m}$ & $824 \mathrm{~ms}$ & $819 \mathrm{~m}$ & 813 & & & \\
\hline $850 \mathrm{~g}$ & - & $849 \mathrm{~ms}$ & 一 & & & 862 & 855 \\
\hline $870_{8}$ & - & - & - & & 869 & & \\
\hline $876 \mathrm{~s}$ & $878 \mathrm{~s}$ & $875 \mathrm{~ms}$ & $876 \mathrm{~m}$ & 880 & & & \\
\hline $915 \mathrm{~m}$ & - & $916 \mathrm{w}$ & - & & & 909 & 906 \\
\hline $927 \mathrm{~m}$ & $932 w$ & $927 \mathrm{w}$ & 927 & 929 & 925 & & \\
\hline & & & & 997 & 998 & 1006 & 987 \\
\hline $1010 \mathrm{w}$ & $1017 \mathrm{sh}$ & $1016 \mathrm{~ms}$ & $1015 \mathrm{vw}$ & 1023 & 1008 & 1015 & 1012 \\
\hline $1036 \mathrm{w}$ & $1036 \mathrm{~m}$ & $1032 \mathrm{~s}$ & $1035 \mathrm{sh}$ & & & & \\
\hline $1048 \mathrm{sh}$ & $1046 \mathrm{w}$ & $1046 \mathrm{~m}$ & $1043 \mathrm{w}$ & 1037 & $\begin{array}{l}1033 \\
1065\end{array}$ & 1033 & $\begin{array}{l}1032 \\
1067\end{array}$ \\
\hline $1080 \mathrm{~m}$ & $1084 \mathrm{w}$ & $1079 \mathrm{~m}$ & $1078 \mathrm{w}$ & 1077 & & 1071 & \\
\hline $1106 \mathrm{w}$ & $1110 \mathrm{vw}$ & $1107 \mathrm{~m}$ & $1106 \mathrm{w}$ & 1106 & & 1091 & 1110 \\
\hline 1127 & - & $1123 \mathrm{~m}$ & - & & 1127 & & \\
\hline $1145 \mathrm{sh}$ & $1148 \mathrm{w}$ & $1145 \mathrm{~m}$ & $1145 \mathrm{w}$ & 1146 & & & \\
\hline $1154 \mathrm{~m}$ & 1162 & $1154 \mathrm{wsh}$ & $1154 w$ & & 1151 & 1155 & 1157 \\
\hline 1231 ส & $1236 \mathrm{~m}$ & 1229 & 1236 & 1225 & 1225 & 1223 & 1229 \\
\hline $1240 \mathrm{vwsh}$ & $1240_{\mathrm{vw}}^{\mathrm{gh}}$ & - & - & 1254 & 1246 & & 1243 \\
\hline & & & & & & 1269 & \\
\hline $1278 \mathrm{~m}$ & $1276 \mathrm{w}$ & $1278 \mathrm{~m}$ & $1278 \mathrm{~m}$ & 1279 & & 1273 & 1282 \\
\hline $1307 \mathrm{msh}$ & - & $1307 \mathrm{w}$ & $1310 \mathrm{~m}$ & & 1290 & 1310 & \\
\hline $1316 \mathrm{~s}$ & $1310 \mathrm{~s}$ & - & - & 1311 & 1314 & & 1321 \\
\hline $1333 \mathrm{w}$ & - & $1335 \mathrm{vw}$ & 一 & 1353 & 1346 & 1348 & 1343 \\
\hline 1342 w & $1348 \mathrm{vw}$ & - & - & & & & \\
\hline- & $1370 \mathrm{~m}$ & $1367 \mathrm{w}$ & $1368 \mathrm{vw}$ & 1367 & 1372 & 1366 & 1371 \\
\hline $1386 \mathrm{~s}$ & $1386 \mathrm{vw}$ & 1387 vw & - & 1389 & 1389 & 1388 & 1387 \\
\hline & & & & 1393 & 1394 & 1393 & 1398 \\
\hline $1442 \mathrm{~m}$ & 1432 & $1442 \mathrm{~s}$ & $1442 \mathrm{~s}$ & 1440 & 1440 & 1442 & 1444 \\
\hline & & & & 1444 & 1448 & 1446 & 1445 \\
\hline $1457 \approx$ & $1451 \mathrm{~m}$ & $1455 \approx$ & $1448 \mathrm{~s}$ & 1458 & 1458 & 1458 & 1458 \\
\hline & & & & 1458 & 1458 & 1458 & 1458 \\
\hline & & & & 1461 & 1461 & 1461 & 1461 \\
\hline $1465 \mathrm{~s}$ & $1467 \mathrm{~m}$ & $1466 \mathrm{sh}$ & $1464 \mathrm{sh}$ & 1462 & 1462 & 1462 & 1462 \\
\hline
\end{tabular}

* Ref. [26]. 
Table 8. Calculated frequencies (in $\mathrm{cm}^{-1}$ ) and potential energy distributions for 3-chloropentene

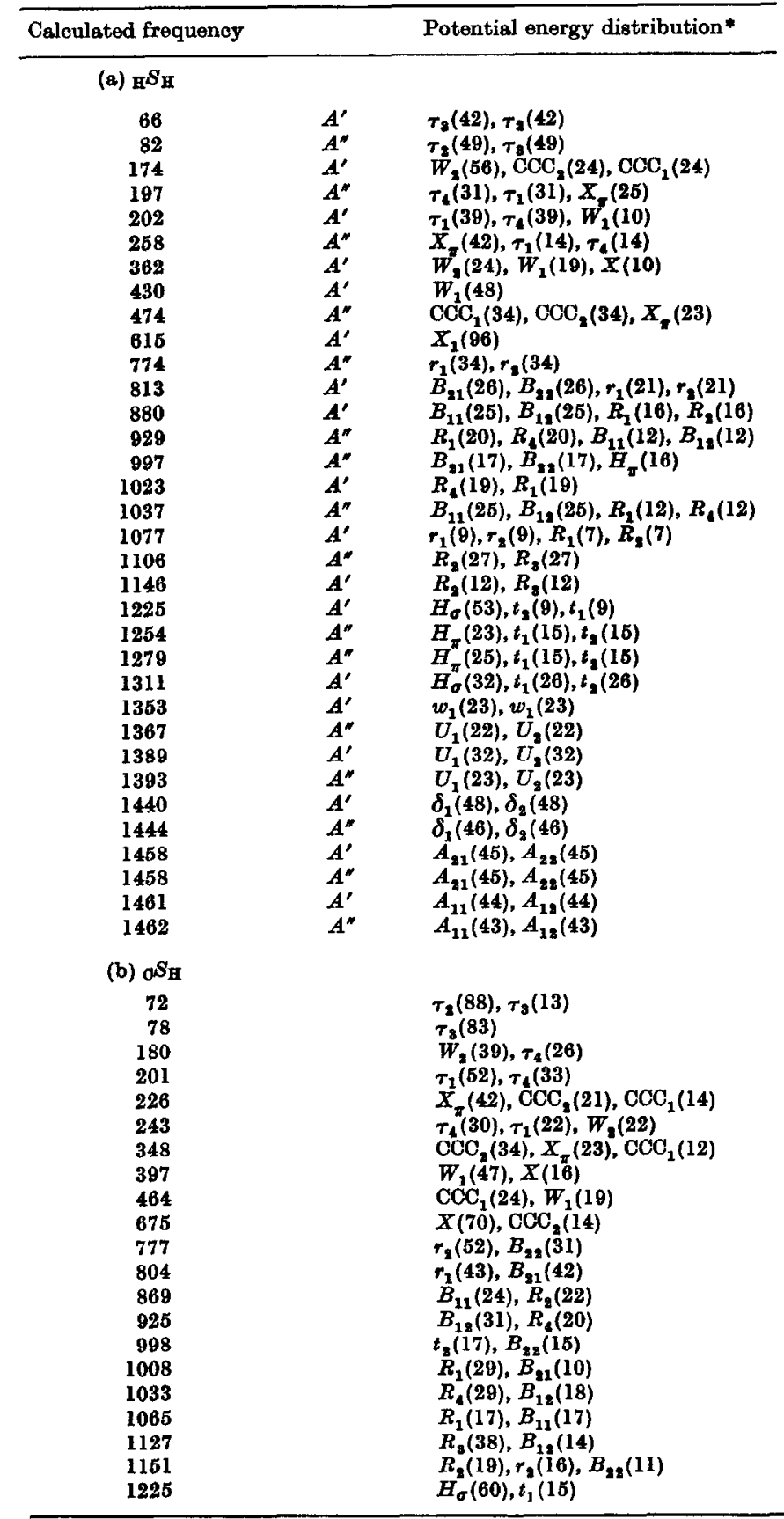

* The torsion coordinates $(\tau)$ are defined in Ref. [1]. Other symmetry coordinates are similar to those defined in Ref. [9]. 
Table 8. (continued)

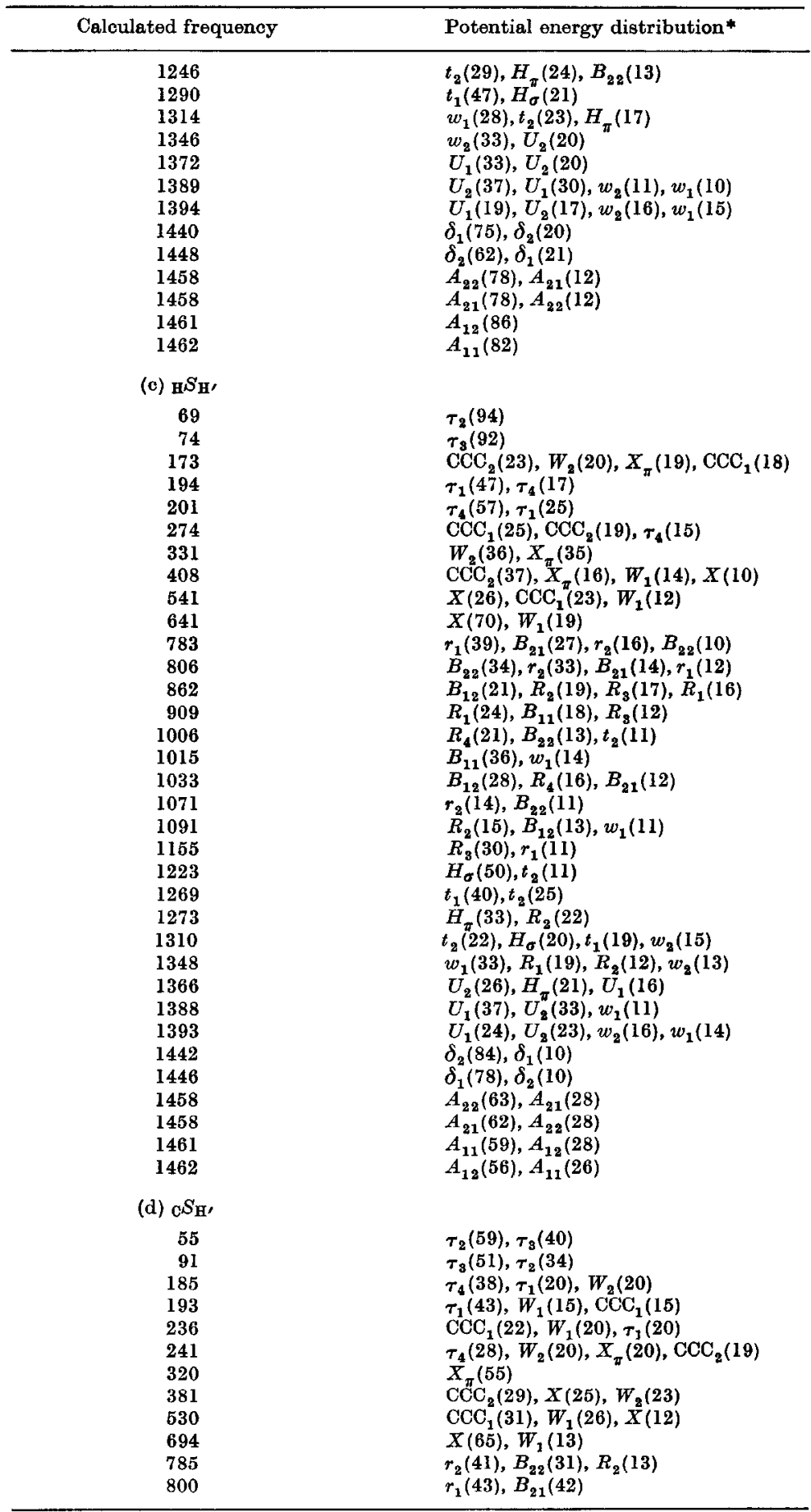


Table 8. (continued)

\begin{tabular}{|c|c|}
\hline Calculated frequency & Potential energy distribution* \\
\hline 855 & $R_{2}(25), B_{22}(18), R_{1}(16)$ \\
\hline 906 & $B_{1 \mathrm{~g}}(24), R_{3}(16), R_{1}(14), R_{4}(11)$ \\
\hline 987 & $R_{4}(20), R_{1}(13), w_{2}(11)$ \\
\hline 1012 & $B_{11}(37), R_{1}(16), H_{\pi}(12)$ \\
\hline 1032 & $R_{4}(27), B_{12}(18)$ \\
\hline 1067 & $B_{12}(18), r_{1}(13), B_{21}(12), t_{1}(12)$ \\
\hline 1110 & $r_{2}(11), H_{\pi}(11)$ \\
\hline 1157 & $R_{3}(29), r_{1}(10)$ \\
\hline 1229 & $H_{\sigma}(66), t_{1}(12)$ \\
\hline 1243 & $t_{3}(35), H_{\pi}(15), B_{38}(14)$ \\
\hline 1282 & $t_{3}(54), H_{\sigma}^{\pi}(17)$ \\
\hline 1321 & $w_{2}(27), t_{2}(23), H_{\pi}(22)$ \\
\hline 1343 & $w_{1}(37), R_{1}(18), R_{2}(16), U_{1}(16)$ \\
\hline 1371 & $U_{2}(44), U_{1}(16), H_{\pi}(15), R_{4}(13)$ \\
\hline 1387 & $U_{1}(50), U_{8}(23), w_{1}(11)$ \\
\hline 1398 & $w_{2}(23), U_{2}(19), w_{1}(14)$ \\
\hline 1444 & $\delta_{2}(82)$ \\
\hline 1445 & $\delta_{1}(82)$ \\
\hline 1458 & $A_{22}(64), A_{21}(27)$ \\
\hline 1458 & $A_{21}(64), A_{22}(27)$ \\
\hline 1461 & $A_{11}(54), A_{12}(34)$ \\
\hline 1462 & $A_{12}(50), A_{11}(31)$ \\
\hline
\end{tabular}

all fundamental vibrational frequencies of the ${ }_{\mathrm{H}} S_{\mathrm{H}}$ isomer. Therefore, at low temperatures an intensity enhancement of vibrations associated with the ${ }_{H} S_{H}$ isomer is expected due to the presence of more molecules in the totally planar zig-zag configuration.

The other $\mathrm{C}-\mathrm{Cl}$ stretching frequencies observed at $634 \mathrm{~cm}^{-1}, 657 \mathrm{~cm}^{-1}$, and $667 \mathrm{~cm}^{-1}$, respectively, are calculated at $641 \mathrm{~cm}^{-1}, 675 \mathrm{~cm}^{-1}$, and $694 \mathrm{~cm}^{-1}$ for the ${ }_{\mathrm{H}} S_{\mathrm{H}^{\prime}}, \mathrm{C}_{\mathrm{H}} S_{\mathrm{H}}$, and ${ }_{\mathrm{C}} S_{\mathrm{H}^{\prime}}$ isomers, respectively (see Tables 7 and 8). The exact geometries assumed by the non-planar forms of 3-chloropentane are presently not known. The works of SCHNEIDER [5] and SÝKORA [27] indicate that, for non-trans conformations, the assumption of a staggered model is not valid. However, these authors have indicated that the staggered approximation is a good model for preferred conformations even when these conformations are non-planar. In light of this we have not addressed ourselves to resolving discrepancies between the observed and calculated frequencies for the ${ }_{\mathrm{C}} S_{\mathrm{H}}$ and $\mathrm{C}_{\mathrm{C}} \mathrm{S}_{\mathrm{H}^{\prime}}$ isomers of 3-chloropentane.

The region of the spectrum below the $\mathrm{C}-\mathrm{Cl}$ stretching vibrations is due to torsional, skeletal, and $\mathrm{C}-\mathrm{C}-\mathrm{Cl}$ bending modes. In each isomer there are four torsional modes. The methyl torsions are predicted to occur at 202 and $197 \mathrm{~cm}^{-1}$ in the ${ }_{\mathrm{H}} S_{\mathrm{H}}$ isomer, at $243 \mathrm{~cm}^{-1}$ and $201 \mathrm{~cm}^{-1}$ in the ${ }_{\mathrm{C}} S_{\mathrm{H}}$ isomer, at 201 and $194 \mathrm{~cm}^{-1}$ in the ${ }_{\mathrm{H}} S_{\mathrm{H}^{\prime}}$ isomer, and at 241 and $193 \mathrm{~cm}^{-1}$ in the ${ }_{\mathrm{O}} S_{\mathrm{H}^{\prime}}$ isomer. The agreement between these torsional modes and the observed bands at 238, 206 and $194 \mathrm{~cm}^{-1}$ supports the value of $0.0775 \mathrm{mdyn}-\AA / \mathrm{rad}^{2}$ for this torsional force constant. The $\mathrm{CH}_{2}-\mathrm{CHCl}$ torsional force constant of $0.0744 \mathrm{mdyn}-\AA / \mathrm{rad}^{2}$ and the interaction torsional force constant of $0.008 \mathrm{mdyn}-\AA / \mathrm{rad}^{2}$ are almost totally responsible for predicting the torsional modes below $100 \mathrm{~cm}^{-1}$. Raman bands are observed at

[27] S. Sx́xora, Collection Czech. Chem. Commun. 33, 3514 (1968). 
approximately 80 and $72 \mathrm{~cm}^{-1}$ for both liquid and solid phase samples of 3-chloropentane. These bands are assigned to the $\mathrm{CH}_{2}-\mathrm{CHCl}$ torsions of ${ }_{\mathrm{H}} S_{\mathrm{H}}$ 3-chloropentane, which are calculated at 82 and $66 \mathrm{~cm}^{-1}$. The excellent correlation which exists between the assignment of this torsion in 2-chlorobutane (observed at $77 \mathrm{~cm}^{-1}$, calculated at $83 \mathrm{~cm}^{-1}$ ) and the assignment in 3-chloropentane confirms the choice of the $\mathrm{CH}_{2}-\mathrm{CHCl}$ torsional force constant.

In the region of the spectrum between $990 \mathrm{~cm}^{-1}$ and $1070 \mathrm{~cm}^{-1}$ three separate bands are observed and either three or four frequencies are predicted for each isomer. Specific assignments in this region are not made. The band observed at $1019 \mathrm{~cm}^{-1} \mathrm{de}-$ oreases in strength when observed in the solid state. Its assignment [26] to the ${ }_{\mathrm{H}} S_{\mathrm{H}}$ isomer is considered questionable. Although three frequencies are predicted in this region for the ${ }_{\mathrm{H}} S_{\mathrm{H}}$ conformation, none of the observed bands in this region displays the enhancement of infrared and Raman intensities which is assumed to be characteristic of the fundamental frequencies of the ${ }_{\mathrm{H}} S_{\mathrm{H}}$ isomer. In the region of 1310$1350 \mathrm{~cm}^{-1}$ the same general features prevail. Any specific assignments would be pure conjecture.

\section{Conclustons}

We have refined a complete valence force field which effectively predicts the normal vibration frequencies of a group of secondary chlorides and related polymers. This force field, which includes elements relating to torsional and intermolecular vibrations, was obtained by using tetrahedral geometry and staggered conformations. The good agreement between observed and calculated frequencies encourages us to believe that this force field can be used in conjunction with energy calculations to give specific answers to questions concerning molecular configuration and conformation in secondary chlorides. The force field also satisfactorily predicts intermolecular frequencies which will be discussed in detail in a subsequent paper [19].

Acknowledgement-This research was supported by National Science Foundation Grant GP. 27717. 